\section{Comparação de estimativas para o auto-relato de condições crônicas entre inquérito domiciliar e telefônico - Campinas (SP), Brasil}

\section{Comparison of estimates for the self- reported chronic conditions among household survey and telephone survey-Campinas (SP), Brazil}

\section{Priscila Maria Stolses Bergamo Francisco' \\ Marilisa Berti de Azevedo Barros' \\ Neuber José Segri" \\ Maria Cecília Goi Porto Alves"' \\ Chester Luiz Galvão Cesar" \\ Deborah Carvalho Malta ${ }^{\mathrm{IV}, \mathrm{v}}$}

'Departamento de Medicina Preventiva e Social da Faculdade de Ciências Médicas da Universidade Estadual de Campinas (UNICAMP) - Campinas (SP) Brasil

"Departamento de Epidemiologia da Faculdade de Saúde Pública da Universidade de São Paulo (USP) - São Paulo (SP), Brasil

"'Instituto de Saúde da Secretaria de Estado da Saúde - São Paulo (SP), Brasil

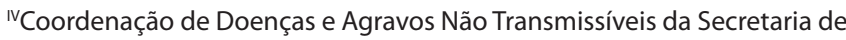
Vigilância em Saúde do Ministério da Saúde - Brasília (DF), Brasil

'Departamento de Enfermagem Materno-Infantil e Saúde Pública da Escola de Enfermagem da Universidade Federal de Minas Gerais (UFMG) - Belo Horizonte (MG), Brasil

Trabalho realizado no Centro Colaborador do Ministério da Saúde em Análise de Situação de Saúde (CCAS) do Departamento de Medicina Preventiva e Social da Faculdade de Ciências Médicas da Universidade Estadual de Campinas (UNICAMP).

Fonte de financiamento: Conselho Nacional de Desenvolvimento Científico e Tecnológico (CNPq

- Processo n 409747/2006-8) e Secretaria Municipal de Saúde de Campinas.

Correspondência: Priscila Maria Stolses Bergamo Francisco - Departamento de Medicina Preventiva e Social da Faculdade de Ciências Médicas da Universidade Estadual de Campinas Caixa Postal 6.111 - CEP: 13083-970 - Campinas (SP) - Brasil - E-mail: primaria@fcm.unicamp.br. Conflito de interesse: nada a declarar.

\section{Resumo}

Objetivo: O objetivo do presente estudo foi comparar as estimativas obtidas por diferentes modalidades de inquérito para condições crônicas auto-referidas em adultos residentes em Campinas (SP) no ano de 2008. Métodos: Foram utilizados os dados do ISACamp, inquérito domiciliar realizado pela Faculdade de Ciências Médicas da Universidade Estadual de Campinas com apoio da Secretaria Municipal de Saúde, e do VIGITEL - Campinas (SP), inquérito telefônico realizado pelo Ministério da Saúde para Vigilância de Fatores de Risco e Proteção para Doenças Crônicas na população adulta (18 anos ou mais). Estimativas do auto-relato de hipertensão arterial, diabetes, osteoporose, asma/bronquite/ enfisema, foram avaliadas e comparadas por meio do teste $t$ de Student para duas amostras independentes. Resultados: Para as estimativas globais, maior prevalência de hipertensão arterial e osteoporose foram verificadas pelo inquérito telefônico. Diabetes e asma/bronquite/enfisema não apresentaram diferenças estatísticas significantes. Na análise segundo variáveis sócio-demográficas, maior prevalência de hipertensão foi obtida pelo VIGITEL para os homens, entre as pessoas de 18 a 59 anos e nos que referiram 9 ou mais anos de estudo. Maior prevalência de osteoporose entre adultos (18 a 59 anos) foi verificada pelo VIGITEL. Em relação à asma/bronquite/enfisema nos idosos, maior prevalência foi observada pelo ISACamp. Conclusão: Exceto para hipertensão arterial, os dados obtidos do inquérito telefônico constituíram uma alternativa rápida para disponibilizar estimativas globais da prevalência das condições estudadas na população adulta residente em Campinas (SP).

Palavras-chave: prevalência; comparações de estimativas; doença crônica; inquéritos epidemiológicos; Brasil; entrevista domiciliar; entrevista por telefone. 


\section{Abstract}

Objective: To compare the estimates obtained by different methods of population-based surveys for self-reported chronic conditions among adults living in Campinas in the year 2008. Methods: Data from ISACamp Survey, conducted by the Faculty of Medical Sciences from Universidade Estadual de Campinas (UNICAMP) with support from the County Health Department and VIGITEL (Campinas), a telephone survey conducted by the Brazilian Ministry of Health toward Surveillance of Risk and Protective Factors for Chronic non-communicable Diseases in the adult population (18 years and over) were analyzed. Estimates of self-reported hypertension, diabetes, osteoporosis, and asthma/bronchitis/emphysema were evaluated and compared by the independent (two-sample) Student's $t$-test. Results: For global estimates, a higher prevalence of hypertension and osteoporosis was ascertained by the telephone survey. Diabetes and asthma/bronchitis/ emphysema results showed no statistically significant differences. According to sociodemographic variables, a higher prevalence of hypertension was obtained by VIGITEL for men, among people aged 18 to 59 years, and those who reported nine or more years of schooling. A higher prevalence of osteoporosis among adults (18 to 59 years) was verified by VIGITEL. Concerning asthma/ bronchitis/emphysema in the elderly, ISACamp survey showed a higher prevalence. Conclusion: Except for the hypertension prevalence, the telephone survey has proven to be a rapid alternative to provide global prevalence estimates of health conditions in the adult population of Campinas.

Keywords: prevalence; comparison of estimates; chronic diseases; health surveys; Brazil; face-to-face interview; telephone interview.

\section{Introdução}

Inquéritos populacionais são instrumentos amplamente utilizados como estudos epidemiológicos para produzir informações necessárias para a formulação e avaliação de políticas sociais e intervenções na área da saúde ${ }^{1}$. Na realização de tais pesquisas, os métodos de coleta de informações foram se aperfeiçoando com o passar do tempo ${ }^{2}$ e as entrevistas realizadas por telefone surgiram como um processo ágil e de menor custo ${ }^{3,4}$.

Neste contexto, a validação de estimativas obtidas por meio de inquéritos telefônicos, demanda a utilização de metodologias específicas para responder questões referentes à representatividade da amostra coletada por meio de cadastros telefônicos, ao impacto que diferentes coberturas de linhas telefônicas residenciais podem causar em tais estimativas, e para dimensionar diferenças entre a população excluída (sem telefone) e aqueles incluídos na amostra em relação aos eventos investigados, embora seja possível utilizar fatores de ponderação ajustando as estimativas para representar a composição sócio-demográfica da população estudada ${ }^{5,6}$.

A comparação de indicadores obtidos por meio de inquéritos domiciliares com inquéritos por telefone vem acontecendo nos Estados Unidos desde que estes últimos passaram a ser utilizados com maior freqüência. O Behavioral Risk Factor Surveillance System (BRFSS) conduzido pelo Centers for Disease Control and Prevention (CDC) desde o início da década de 1980, com o objetivo de coletar dados sobre fatores de risco para a população adulta, vem utilizando essa metodologia e apresentando resultados válidos ${ }^{5}$.

As doenças crônicas não transmissíveis (DCNT) têm se destacado como as principais causas de morbimortalidade nas últimas décadas em todo o mundo ${ }^{7}$. Inquéritos populacionais periódicos permitem o monitoramento da prevalência dessas condições e de seus principais fatores de risco; também possibilitam avaliações do impacto 
de intervenções em ampla escala, visando a prevenção primária destas doenças ${ }^{8}$.

A aplicação de questionários para obtenção da informação auto-referida é uma estratégia de menor custo, acessível e rápida para estimar prevalências, apesar de estar sujeita a erros de classificação. No entanto, a acurácia da informação coletada por auto-relato depende das características do questionário, da forma de aplicação, da capacitação dos entrevistadores e da população à qual ele é aplicado 9 .

Os inquéritos populacionais devem constituir parte integrante do sistema nacional de informações em saúde e são essenciais para monitorar condições e situação de saúde com indicadores não disponíveis nas bases de dados secundários dos sistemas de informações ${ }^{10}$. Portanto, a avaliação de informações oriundas de diferentes modalidades de inquérito torna-se necessária para o aprimoramento do sistema.

O objetivo do presente estudo foi comparar as estimativas de prevalência obtidas por diferentes modalidades de inquérito para hipertensão arterial, diabetes, osteoporose e asma/bronquite/enfisema em adultos residentes em Campinas.

\section{Métodos}

Os dados utilizados neste estudo foram obtidos do Inquérito de Saúde no município de Campinas (ISACamp), realizado pelo Centro Colaborador do Ministério da Saúde em Análise de Situação de Saúde (CCAS) do Departamento de Medicina Preventiva e Social da Faculdade de Ciências Médicas da Universidade Estadual de Campinas (UNICAMP) e da Vigilância de Fatores de Risco e Proteção para Doenças Crônicas por Inquérito Telefônico (VIGITEL Campinas) realizado pela Secretaria de Vigilância em Saúde (SVS) do Ministério da Saúde, ambos no ano de 2008.

\section{ISACamp}

No período de abril de 2001 e março de 2002, foi realizado o Inquérito de Saúde no
Estado de São Paulo (ISA-SP), um estudo de corte transversal desenhado para analisar condições de vida, situação de saúde e uso de serviços de saúde em diferentes áreas do Estado de São Paulo, por meio de entrevistas domiciliares ${ }^{11}$. O projeto aprovado pelo Programa de Políticas Públicas da FAPESP abrangeu quatro áreas do Estado, duas no interior - nos municípios de Botucatu e Campinas - e duas na Região da Grande São Paulo - na Subprefeitura do Butantã (município de São Paulo) e em três municípios da região sudoeste da Grande São Paulo, Taboão da Serra, Embu e Itapecerica da Serra. Tendo como base essa pesquisa, realizou-se novo inquérito de saúde no município de Campinas (ISACamp) no ano de 2008, com revisão dos conteúdos e do questionário aplicado.

O ISACamp foi desenhado para analisar os perfis do estado de saúde, estilo de vida (fatores de risco para doenças crônicas) e uso de serviços de saúde de diferentes segmentos sociais da população, avaliar o grau de equidade/desigualdade social da população do município, e, especificamente, monitorar os indicadores de saúde e desigualdades sociais em saúde.

Os dados foram obtidos por meio de inquérito domiciliar de base populacional sendo que, para a população residente não-institucionalizada com idade de 18 anos ou mais, $90,4 \%$ das entrevistas foram realizadas no ano de 2008. Como objetivo da pesquisa, fixou-se o estudo de aspectos referentes a três subgrupos dessa população: adolescentes, adultos e idosos. Dessa forma, a população foi divida em três estratos que constituíram domínios de estudo e para os quais foram sorteadas amostras independentes.

A amostra do inquérito foi obtida por procedimentos de amostragem probabilística, estratificada, por conglomerados e em dois estágios: setor censitário e domicílio. No primeiro estágio, foram sorteados 50 setores censitários com probabilidade proporcional ao número de domicílios. O sorteio foi sistemático, ordenando-se os setores pelo percentual de chefes de 
família que possuíam nível universitário, produzindo uma estratificação implícita por escolaridade do chefe de família.

$\mathrm{O}$ número de pessoas para compor a amostra foi obtido considerando-se a situação correspondente à máxima variabilidade para a frequência dos eventos estudados $(\mathrm{p}=0,50)$, um coeficiente de confiança de 95\% na determinação dos intervalos de confiança $(\mathrm{z}=1,96)$, erro de amostragem entre 4 e 5 pontos percentuais e efeito de delineamento igual a 2, totalizando 1.000 indivíduos em cada domínio de idade, a saber: adolescentes (10 a 19 anos), adultos (20 a 59 anos) e idosos (60 anos ou mais). Esperando-se uma taxa de $\mathbf{8 0 \%}$ de cobertura e resposta, o tamanho da amostra foi corrigido para 1.250. Para atingir esse tamanho de amostra em cada domínio, após atualização em campo dos mapas dos setores sorteados e elaboração da listagem de endereços, foram selecionados, de forma independente, 2.150, 700 e 3.900 domicílios para adolescentes, adultos e idosos, respectivamente. Em cada domicílio, entrevistadores treinados e supervisionados obtiveram as informações de todos os moradores da faixa etária selecionada para aquele domicílio.

O questionário aplicado, previamente testado em um estudo piloto, foi estruturado com a maioria das questões fechadas, com alternativas pré-definidas e organizado em blocos, segundo áreas temáticas: estado de saúde, uso de serviços de saúde, comportamentos relacionados à saúde e condições socioeconômicas. Os dados foram digitados em banco de dados com o uso do software EpiData versão 3.1, o qual também foi utilizado para avaliar a consistência das informações obtidas. Em seguida, utilizou-se o programa Stata 11 para o cálculo dos pesos amostrais que levam em conta o delineamento complexo, a nãoresposta e a pós-estratificação.

\section{VIGITEL}

O sistema VIGITEL foi implantado no país em 2006, objetivando monitorar continuamente a frequência e distribuição de fatores de risco e proteção para doenças crônicas nas capitais de todos os Estados brasileiros e no Distrito Federal, por meio da realização de entrevistas telefônicas assistidas por computador em amostras probabilísticas da população adulta (18 anos ou mais), residente em domicílios servidos por linhas de telefone fixo residencial de cada cidade ${ }^{12}$.

O município de Campinas foi contemplado pelo Ministério da Saúde para a realização do inquérito por telefone (VIGITEL - Campinas), visto que no mesmo ano seria realizado o ISACamp.

Para a coleta das informações, utilizou-se amostragem probabilística da população adulta realizada em duas etapas: sorteio das linhas telefônicas e sorteio do morador do domicílio a ser entrevistado. Os procedimentos adotados visaram obter amostras de adultos residentes em domicílios servidos por pelo menos uma linha telefônica fixa no ano ${ }^{12}$. O tamanho amostral mínimo de 2.000 indivíduos com 18 anos ou mais de idade permite estimar, com coeficiente de confiança de $95 \%$ e erro máximo de cerca de $2 \%$, a frequência de qualquer fator de risco na população adulta ${ }^{13}$.

Inicialmente, foi realizado o sorteio sistemático de 5.000 linhas telefônicas, a partir do cadastro eletrônico de linhas residenciais fixas da empresa Telefônica, ordenado por prefixo, levando à estratificação implícita por região do município. Em seguida, as linhas sorteadas foram ressorteadas e divididas em 25 réplicas de 200 linhas, cada réplica reproduzindo a mesma proporção de linhas por prefixo telefônico. A divisão da amostra em réplicas torna-se necessária em função da dificuldade em se estimar previamente a proporção de linhas do cadastro elegíveis para o sistema (linhas residenciais ativas) e, portanto, o total de linhas a ser sorteado para obtenção de 2.000 entrevistas ${ }^{12}$.

Em Campinas, foram realizadas ligações para 4.800 linhas telefônicas distribuídas em 24 réplicas, identificando-se 2.773 linhas elegíveis. Para cada linha elegível, uma vez obtida a aquiescência dos seus usuários em participar do sistema, 
procedeu-se à enumeração dos indivíduos com 18 anos ou mais que residiam no domicílio e, a seguir, o sorteio de um deles para ser entrevistado ${ }^{12}$.

O questionário foi construído de modo a viabilizar a opção do sistema pela realização de entrevistas telefônicas feitas com o emprego de computadores - entrevistas cujas perguntas são lidas diretamente da tela do monitor de vídeo e as respostas são instantaneamente digitadas. As perguntas do VIGITEL abordaram características demográficas e socioeconômicas dos indivíduos, informações sobre hábito alimentar e de atividade física associadas à ocorrência de DCNT, peso e altura, frequência de consumo de cigarros e de bebidas alcoólicas, autoavaliação do estado de saúde do entrevistado e referência a diagnóstico médico anterior de algumas doenças ${ }^{14}$.

Para diminuir o vício gerado pela não cobertura universal de telefonia fixa, foram utilizados fatores de ponderação. $\mathrm{O}$ peso final atribuído a cada indivíduo entrevistado é o resultado da multiplicação dos seguintes fatores: o inverso do número de linhas telefônicas no domicílio do entrevistado, o número de adultos no domicílio do entrevistado, e a razão entre a frequência relativa de indivíduos na amostra do Censo-2000 e a frequência relativa na amostra estudada (já incorporando os dois primeiros fatores); considerando-se 36 estratos segundo sexo (masculino e feminino), faixa etária (18-24, 25-34, 35-44, $45-54,55-64$ e 65 ou mais anos de idade) e nível de escolaridade (0-8, 9-11 e 12 ou mais anos de estudo) $)^{12}$.

\section{Presente estudo}

Para realização do presente estudo, foram incluídos 2.636 adultos (18 anos ou mais) não institucionalizados, residentes em área urbana no município de Campinas no ano de 2008, amostrados pelo ISACamp-2008 e 2.015 indivíduos de 18 anos ou mais entrevistados pelo VIGITEL - Campinas.
Nos bancos de dados analisados, o ajuste de pós-estratificação considerou a composição sóciodemográfica, segundo a distribuição censitária do ano 2000, utilizando nos dados do ISACamp a mesma estratégia de ponderação usada pelo VIGITEL e descrita anteriormente ${ }^{12}$.

Sexo, faixa etária e escolaridade foram as variáveis utilizadas para caracterizar a população estudada. As variáveis analisadas para avaliar a comparabilidade das estimativas foram hipertensão arterial, diabetes, osteoporose e asma/bronquite/enfisema. No ISACamp, as informações foram obtidas por meio da seguinte pergunta: "Algum médico ou profissional de saúde já disse que você tem alguma das seguintes doenças?" As categorias de resposta disponíveis eram "sim", "não" e "não sabe". Pelo VIGITEL, as questões utilizadas na coleta destas condições/doenças foram: "Algum médico já lhe disse que o(a) sr(a) tem pressão alta? E diabetes? E osteoporose (doença/fraqueza dos ossos)? E doenças como asma, bronquite asmática, bronquite crônica ou enfisema?" Neste caso, as possibilidades de resposta eram "sim", "não" e "não lembra".

Neste estudo, as diferenças entre os inquéritos foram estimadas utilizando-se um arquivo de dados combinado. Para tanto, as variáveis de ambos os bancos de dados foram renomeadas e, em seguida, classificadas com valores iguais para a mesma categoria de resposta, viabilizando as análises de comparação ${ }^{15}$.

Foram calculadas as prevalências e intervalos de confiança de $95 \%$, para as variáveis selecionadas obtidas de ambas as modalidades de inquérito. Para as doenças crônicas anteriormente mencionadas, também foram calculadas as estimativas segundo sexo, faixa etária e escolaridade. A diferença estatística entre as estimativas do ISACamp e VIGITEL - Campinas foi verificada pelo teste $t$ de Student para duas amostras independentes ${ }^{15}$. Nas análises foram considerados os aspectos do delineamento amostral complexo por meio da utilização do programa Stata $11^{6}$. 
$\mathrm{O}$ projeto de pesquisa do inquérito domiciliar foi aprovado pela Comissão de Ética da Universidade Estadual de Campinas (Parecer $\mathrm{n}^{\circ}$ 079/2007) e o projeto de implantação do VIGITEL foi aprovado pelo Comitê Nacional de Ética em Pesquisa do Ministério da Saúde (CONEP 13081/2008).

\section{Resultados}

A média de idade dos adultos entrevistados pelo ISACamp $(\mathrm{n}=2.636)$ foi de 40,1 anos (IC 95\%: 39,0-41,3). Entre os entrevistados pelo VIGITEL - Campinas $(\mathrm{n}=2.015)$, a média de idade foi de 39,9 anos (IC 95\%: 38,9-40,9). As taxas de resposta obtidas foram de 85,6 e de $72,7 \%$ (entrevistas realizadas/linhas elegíveis) por meio do inquérito domiciliar e inquérito telefônico, respectivamente.

As características sóciodemográficas dos participantes de ambos os inquéritos estão apresentadas na Tabela 1. Nas análises segundo sexo, faixa etária e escolaridade observam-se semelhanças, em função do recálculo da ponderação do banco de dados do ISACamp, segundo a estrutura sóciodemográfica utilizada pelo VIGITEL. Dentre os entrevistados, mais da metade eram mulheres e predominaram os adultos jovens.

Considerando-se a prevalência das condições crônicas selecionadas neste estudo na população de 18 anos ou mais, foi detectada maior prevalência de hipertensão arterial e osteoporose pelo inquérito telefônico. Diabetes e asma/bronquite/enfisema apresentaram prevalências similares (Tabela 2).

Na Tabela 3, são apresentadas as prevalências segundo variáveis sociodemográficas. Dados do inquérito telefônico apontaram maior prevalência de hipertensão arterial entre os homens $(\mathrm{p}=0,023)$, nas pessoas de 18 a 59 anos $(\mathrm{p}=0,005)$ e nos que referiram 9 a 11 anos $(p=0,029)$ e 12 ou mais $(\mathrm{p}=0,014)$ anos de estudo. Da mesma forma, a prevalência de osteoporose foi maior para a faixa de 18 a 59 anos $(\mathrm{p}=0,004)$. Já em relação à asma/bronquite/enfisema nos idosos, maior prevalência $(\mathrm{p}<0,001)$ foi verificada pelo inquérito domiciliar.

Tabela 1. Distribuição percentual da população adulta (18 anos ou mais), segundo características sociodemográficas. ISACamp e VIGITEL. Campinas, 2008

Table 1. Frequency distribution of adult population ( $\geq 18$ year of age), according to sociodemographic characteristics. ISACamp and VIGITEL. Campinas, 2008

\begin{tabular}{lcccc}
\hline \multirow{2}{*}{ Variáveis e categorias } & \multicolumn{2}{c}{ ISACamp } & \multicolumn{2}{c}{ VIGITEL } \\
\cline { 2 - 5 } & $\mathrm{n}$ & $(\%)^{1}$ & $\mathrm{n}$ & $(\%)^{1}$ \\
\hline Sexo & & & & \\
$\quad$ Masculino & 1.141 & $47,9(45,6-50,2)$ & 851 & $47,9(44,8-51,0)$ \\
$\quad$ Feminino & 1.495 & $52,1(49,8-54,4)$ & 1.164 & $52,1(49,0-55,2)$ \\
Faixa etária (anos) & & & & \\
18 a 24 & 321 & $19,4(17,0-21,8)$ & 240 & $19,4(16,3-22,5)$ \\
25 a 34 & 273 & $24,5(21,1-27,9)$ & 379 & $24,5(21,6-27,4)$ \\
35 a 44 & 222 & $21,8(19,2-24,5)$ & 471 & $21,8(19,6-24,1)$ \\
45 a 54 & 206 & $15,6(13,2-17,9)$ & 360 & $15,6(13,7-17,4)$ \\
55 a 64 & 570 & $9,5(7,9-11,2)$ & 275 & $9,5(8,2-10,9)$ \\
65 e mais & 1.044 & $9,1(7,6-10,7)$ & 290 & $9,1(7,9-10,4)$ \\
Escolaridade & & & & \\
0 a 8 anos & 1.568 & $54,5(48,1-60,9)$ & 711 & $54,6(51,6-57,5)$ \\
9 a 11 anos & 528 & $25,7(22,5-28,9)$ & 703 & $25,6(23,4-27,8)$ \\
12 anos ou mais & 540 & $19,8(13,5-26,1)$ & 592 & $19,8(18,0-21,7)$ \\
\hline
\end{tabular}

$\mathrm{n}$ - número de indivíduos na amostra não ponderada; ${ }^{1}$ prevalência na amostra ponderada

$n$-number of individuals in the unweighted sample; prevalence in the weighted sample 
Tabela 2. Prevalência de condições crônicas na população adulta ( 18 anos ou mais). ISACamp e VIGITEL. Campinas, 2008 Table 2. Prevalence of chronic conditions in the adult populations ( $\geq 18$ year of age). ISACamp and VIGITEL. Campinas, 2008

\begin{tabular}{|c|c|c|c|c|c|c|c|}
\hline \multirow{2}{*}{ Variáveis e Categorias } & \multicolumn{2}{|r|}{ ISACamp } & \multicolumn{2}{|r|}{ VIGITEL } & \multicolumn{2}{|c|}{ Diferença estimada } & \multirow{2}{*}{$p^{*}$} \\
\hline & $\mathrm{n}$ & $\%^{1}$ (IC 95\%) & $\mathrm{n}$ & $\%^{1}$ (IC 95\%) & $\%$ & (IC 95\%) & \\
\hline \multicolumn{8}{|l|}{ Condições crônicas ${ }^{1}$} \\
\hline Hipertensão & 959 & $18,6(16,8-20,4)$ & 533 & $22,3(20,0-24,6)$ & $-0,038$ & $(-0,067--0,009)$ & 0,011 \\
\hline Diabetes & 369 & $5,9(4,6-7,2)$ & 140 & $6,0(4,8-7,2)$ & $-0,001$ & $(-0,019-0,017)$ & 0,944 \\
\hline Osteoporose & 241 & $2,7(2,0-3,3)$ & 102 & $3,8(2,9-4,7)$ & $-0,011$ & $(-0,022--0,006)$ & 0,039 \\
\hline $\begin{array}{l}\text { Asma/bronquite } \\
\text { ou enfisema }\end{array}$ & 136 & $3,9(2,8-5,0)$ & 63 & $3,3(2,2-4,3)$ & 0,006 & $(-0,009-0,021)$ & 0,434 \\
\hline
\end{tabular}

*Diferenças entre o ISACamp e o VIGITEL - Campinas, baseada no teste $t$ de Student para duas amostras independentes;' prevalência na amostra ponderada *Differences between ISACamp and VIGITEL Campinas, based on Student's t-test for two independent samples;' prevalence in the weighted sample

Tabela 3. Prevalência de condições crônicas na população adulta ( 18 anos ou mais), segundo variáveis sociodemográficas. ISACamp e VIGITEL. Campinas, 2008

Table 3. Prevalence of chronic conditions in the adult population ( $\geq 18$ year of age), according sociodemographic variables. ISACamp and VIGITEL. Campinas, 2008

\begin{tabular}{|c|c|c|c|c|c|c|c|c|}
\hline & \multicolumn{2}{|c|}{ Hipertensão arterial (\%) ${ }^{1}$} & \multicolumn{2}{|c|}{ Diabetes $(\%)^{1}$} & \multicolumn{2}{|c|}{ Osteoporose (\%) ${ }^{1}$} & \multicolumn{2}{|c|}{$\begin{array}{c}\text { Asma/bronquite/enfisema } \\
(\%)^{1}\end{array}$} \\
\hline & ISACamp & VIGITEL & ISACamp & VIGITEL & ISACamp & VIGITEL & ISACamp & VIGITEL \\
\hline \multicolumn{9}{|l|}{ Sexo } \\
\hline \multirow[t]{2}{*}{ Masculino } & 15,5 & 20,4 & 4,9 & 4,9 & 0,9 & 1,4 & 3,4 & 2,1 \\
\hline & $(13,0-18,0)$ & $(17,0-23,8)^{*}$ & $(3,4-6,5)$ & $(3,3-6,5)$ & $(0,4-1,5)$ & $(0,5-2,3)$ & $(2,0-4,7)$ & $(1,0-3,2)$ \\
\hline \multirow[t]{2}{*}{ Feminino } & 21,4 & 24,1 & 6,8 & 6,9 & 4,3 & 6,0 & 4,3 & 4,4 \\
\hline & $(18,9-23,8)$ & $(21,0-27,2)$ & $(5,2-8,5)$ & $(5,2-8,7)$ & $(3,2-5,3)$ & $(4,6-7,4)$ & $(2,6-6,0)$ & $(2,7-6,1)$ \\
\hline
\end{tabular}

\begin{tabular}{|c|c|c|c|c|c|c|c|c|}
\hline \multicolumn{9}{|l|}{$\begin{array}{l}\text { Faixa etária } \\
\text { (anos) }\end{array}$} \\
\hline 18 a 59 & $\begin{array}{c}13,0 \\
(11,2-14,8)\end{array}$ & $\begin{array}{c}17,2 \\
(14,8-19,6)^{*}\end{array}$ & $\begin{array}{c}3,3 \\
(1,9-4,7)\end{array}$ & $\begin{array}{c}4,0 \\
(2,9-5,2)\end{array}$ & $\begin{array}{c}0,7 \\
(0,2-1,2)\end{array}$ & $\begin{array}{c}1,6 \\
(0,9-2,2)^{*}\end{array}$ & $\begin{array}{c}3,5 \\
(2,3-4,8)\end{array}$ & $\begin{array}{c}3,5 \\
(2,3-4,6)\end{array}$ \\
\hline 60 ou mais & $\begin{array}{c}53,4 \\
(50,1-56,6)\end{array}$ & $\begin{array}{c}54,8 \\
(49,0-60,5)\end{array}$ & $\begin{array}{c}21,9 \\
(19,5-24,2)\end{array}$ & $\begin{array}{c}18,2 \\
(13,8-22,6)\end{array}$ & $\begin{array}{c}14,8 \\
(12,3-17,2)\end{array}$ & $\begin{array}{c}17,8 \\
(13,4-22,2)\end{array}$ & $\begin{array}{c}6,1 \\
(4,3-7,8)\end{array}$ & $\begin{array}{c}2,0 \\
(0,6-3,4)^{*}\end{array}$ \\
\hline \multicolumn{9}{|l|}{ Escolaridade } \\
\hline 0 a 8 anos & $\begin{array}{c}25,7 \\
(22,4-29,0)\end{array}$ & $\begin{array}{c}28,3 \\
(24,3-32,2)\end{array}$ & $\begin{array}{c}8,4 \\
(6,1-10,7)\end{array}$ & $\begin{array}{c}8,2 \\
(6,1-10,2)\end{array}$ & $\begin{array}{c}4,1 \\
(2,9-5,3)\end{array}$ & $\begin{array}{c}5,4 \\
(3,9-6,9)\end{array}$ & $\begin{array}{c}4,2 \\
(2,5-5,8)\end{array}$ & $\begin{array}{c}3,8 \\
(2,1-5,5)\end{array}$ \\
\hline 9 a 11 anos & $\begin{array}{c}9,6 \\
(6,3-12,9)\end{array}$ & $\begin{array}{c}14,3 \\
(11,7-17,0)^{*}\end{array}$ & $\begin{array}{c}2,6 \\
(1,0-4,2)\end{array}$ & $\begin{array}{c}3,0 \\
(1,6-4,3)\end{array}$ & $\begin{array}{c}0,8 \\
(0,2-1,4)\end{array}$ & $\begin{array}{c}1,7 \\
(0,8-2,6)\end{array}$ & $\begin{array}{c}3,5 \\
(1,6-5,4)\end{array}$ & $\begin{array}{c}3,0 \\
(1,6-4,3)\end{array}$ \\
\hline $\begin{array}{l}12 \text { anos ou } \\
\text { mais }\end{array}$ & $\begin{array}{c}10,5 \\
(7,5-13,5)\end{array}$ & $\begin{array}{c}16,1 \\
(12,8-19,5)^{*}\end{array}$ & $\begin{array}{c}3,3 \\
(1,8-4,8)\end{array}$ & $\begin{array}{c}3,7 \\
(2,0-5,4)\end{array}$ & $\begin{array}{c}1,1 \\
(0,5-1,7)\end{array}$ & $\begin{array}{c}1,8 \\
(0,8-2,9)\end{array}$ & $\begin{array}{c}3,6 \\
(1,6-5,6)\end{array}$ & $\begin{array}{c}2,2 \\
(0,9-3,5)\end{array}$ \\
\hline
\end{tabular}

${ }^{*}$ Diferença significativa $(p<0,05)$ entre o ISACamp e o VIGITEL-Campinas, baseada no teste $t$ de Student para duas amostras independentes; ${ }^{1}$ prevalência na amostra ponderada

*Significant difference $(p<0.05)$ and between ISACamp and VIGITEL-Campinas, based on Student's t test for two independent samples; 'prevalence in the weighted sample

\section{Discussão}

O ISACamp e o VIGITEL são inquéritos populacionais com diferentes delineamentos amostrais e que usaram diferentes métodos para coletar os dados, mas possuem várias similaridades. Ambos foram conduzidos no decorrer do ano de 2008, reduzindo o viés sazonal e utilizaram algumas questões análogas para várias medidas, como para as condições crônicas investigadas. Neste estudo, os inquéritos domiciliar e telefônico apresentaram resultados globais semelhantes para a prevalência de diabetes e de bronquite/ asma/enfisema autorreferidos. Maiores 
prevalências de hipertensão arterial e osteoporose foram estimadas pelo inquérito telefônico.

Os inquéritos populacionais constituem fonte fundamental de informações em saúde ${ }^{1,10}$ e torna-se importante ampliar o conhecimento sobre as estratégias e instrumentos empregados, como as entrevistas face a face, os inquéritos telefônicos, questionários autoaplicados enviados por correio, dentre outros. Diversos autores consideram o inquérito face a face como o método de escolha, ou padrão ouro, na coleta de dados, entretanto, estudos têm demonstrado a similaridade com o uso de outras estratégias ${ }^{5,17}$.

Estudos apontam que a acurácia da informação referida de morbidade pode variar conforme a patologia pesquisada, a presença de comorbidades, a gravidade da doença e características sociais e demográficas do respondente ${ }^{18-22}$. O reconhecimento da doença pelo indivíduo depende ainda do grau de percepção de sinais e sintomas, do acesso aos serviços médicos e aos testes diagnósticos, bem como do tipo e da qualidade das orientações obtidas dos profissionais de saúde.

A hipertensão arterial é considerada um dos mais importantes fatores de risco para doenças não transmissíveis ${ }^{9} \mathrm{e}$ é considerada uma morbidade para outras. A validade da informação autorreferida para hipertensão arterial é amplamente descrita na literatura ${ }^{9,18,19,21,23,24}$. Além disso, para o sexo feminino, idade mais avançada, sobrepeso e uso de serviços de saúde são apontados como fatores que aumentam a validade do auto-relato de hipertensão $0^{9,21,23,24}$. No que se refere à comparação de estimativas da prevalência da doença em inquéritos, Nelson et al. ${ }^{5}$ utilizando dados do National Health Interwiew Survey (NHIS) e do Behavioral Risk Factor Surveillance System (BRFSS), verificaram diferenças significantes para a população de 18 a 34 anos (maior prevalência obtida pelo inquérito telefônico) e nos maiores de 55 anos (prevalência superior verificada pelo inquérito domiciliar). Os autores não encontraram diferenças segundo gênero ou escolaridade.

Entre as doenças crônicas não transmissíveis, o diabetes se destaca como importante causa de morbimortalidade, principalmente entre os idosos. Para Cricelli et al. ${ }^{18} \mathrm{e}$ Okura et al. ${ }^{19}$, a resposta auto-referida para diabetes apresenta alta acurácia. No entanto, Lima-Costa et al. ${ }^{22}$, investigando a validade da informação auto-referida entre os idosos que participaram do projeto Bambuí, registraram sensibilidade de 57,1\% e alta especificidade $(96,0 \%)$ e observaram que a escolaridade e o uso de serviços de saúde foram determinantes na capacidade do idoso informar sua condição de diabético corretamente. Estudo holandês realizado com indivíduos com idade de 18 anos ou mais, encontrou sensibilidade e especificidade de $58,9 \%$ e $99,4 \%$ respectivamente para o diabetes auto-referido ${ }^{20}$. Na avaliação de dados de 1997 do NHIS e do BRFSS, foram encontradas diferenças nas estimativas da prevalência de diabetes entre as pessoas de 55 anos ou mais e também entre os homens (maiores prevalências pelo NHIS) ${ }^{5}$. Já na análise de dados de 2004 do BRFSS, do NHIS e do National Health and Nutrition Examination Survey (NHANES), não houve diferença nas estimativas globais nem nas estimativas segundo sexo, idade e escolaridade ${ }^{17}$.

Embora a osteoporose seja a doença osteometabólica mais comum, o número de estudos sobre a prevalência da doença é pequeno no país ${ }^{25}$. Para Martini et al. ${ }^{26}$, diferenças raciais e genéticas, antropométricas, sócio-culturais e econômicas, nutricionais, além da utilização de serviços de saúde nos vários países, são fatores que poderiam explicar as divergências na incidência e na prevalência da doença. Neste estudo, foi possível observar a importância da doença principalmente entre as mulheres e nas faixas etárias mais avançadas, conforme verificado em outros estudos ${ }^{25,26}$. Apesar de não ter sido localizado estudo sobre a validação de osteoporose autoreferida, vale ressaltar que condições com os critérios diagnósticos mais precisos são 
mais propensas a relatos mais fidedignos do que aquelas com critérios menos claros e/ou patologias menos incapacitantes. Não foi localizado estudo que tivesse comparado estimativas de prevalência de osteoporose com dados de inquérito domiciliar e telefônico.

Em relação às condições respiratórias, alguns autores chamam a atenção para a validade de auto-referência de sintomas respiratórios, asma e doença pulmonar obstrutiva crônica em inquéritos, com sensibilidade e especificidade adequadas em screenings populacionais, podendo refletir indiretamente a prevalência real na comunidade $^{27,28}$. Na comparação das estimativas da prevalência de asma auto-referida, Fahimi et al. ${ }^{17}$ encontraram diferenças globais e segundo características sócio-demográficas a saber: idade, sexo, cor/raça e escolaridade $(\mathrm{p}<0,001)$. Dados do inquérito telefônico (BRFSS) apresentaram maiores prevalências em relação ao inquérito domiciliar (NHIS).

Estimativas de doenças crônicas baseadas na morbidade auto-referida, apresentam como vantagens a rapidez na obtenção da informação e o baixo custo, principalmente quando obtidas por inquérito telefônico, tornando viável sua utilização em grandes populações. No entanto, entre as limitações deste tipo de pesquisa, deve-se considerar que essas informações estão sujeitas a vieses, já que dependem do conhecimento do respondente quanto à informação de interesse, de sua capacidade de recordá-la e do seu desejo de informar. Além disto, a doença pode não ter sido diagnosticada, ou seja, a prevalência da morbidade ou condição crônica investigada pode ser subestimada ${ }^{29,30}$.

Nas últimas décadas, os levantamentos de base populacional vêm se destacando entre as pesquisas pela sua capacidade de mensurar fatores de risco modificáveis. Os resultados deste estudo evidenciaram o potencial dos inquéritos na obtenção de informações sobre as condições crônicas estudadas. A semelhança dos resultados destaca ainda a adequação do instrumento utilizado, já que as duas pesquisas foram realizadas mediante delineamentos amostrais distintos. Chrestani, Santos e Matijasevich $^{9}$ apontam a necessidade da padronização da pergunta na comparação de informações nos diferentes inquéritos. Além disso, a ordem das perguntas pode influenciar os resultados ${ }^{5}$.

Dentre as limitações do inquérito telefônico, deve-se considerar o fato da amostra se restringir àqueles que possuem telefone fixo. No entanto, no município de Campinas, as coberturas telefônicas são elevadas, possibilitando estimativas populacionais adequadas, conforme apontado por Bernal e Silva ${ }^{6}$. Além disto, o uso de fatores de expansão possibilita a redução do viés, buscando aproximar a amostra do estudo da população total ${ }^{6}$. Dentre as vantagens desta modalidade de inquérito, destaca-se a agilidade do sistema para subsidiar programas e políticas de saúde, bem como seu baixo custo. Já os inquéritos domiciliares são uma modalidade de pesquisa de maior custo em função do treinamento dos entrevistadores e do deslocamento para realização das entrevistas, no entanto, abrangem o conjunto da população com e sem telefone, possibilitam verificar in loco as condições de vida do entrevistado, maior fidedignidade da resposta obtida e permitem entrevistas mais longas.

A importância deste estudo reside no fato de ser um dos primeiros no país e mesmo na literatura internacional, a comparar informações oriundas de diferentes modalidades de inquérito que utilizaram diferentes técnicas de coleta de dados e que possuem delineamentos amostrais distintos, mas que, no entanto, foram conduzidos durante o mesmo período de tempo e local, e utilizaram questões semelhantes para obtenção de informações sobre as condições crônicas avaliadas, tornando-as passíveis de comparação.

Para Barros ${ }^{10}$, inquéritos de saúde locais constituem espaços e oportunidades de experimentação e validação de instrumentos. A utilização de uma ou outra modalidade 
de inquérito dependerá da situação para a qual as estimativas serão utilizadas.

\section{Conclusão}

Existem limites na comparação de inquéritos como o desenho amostral, perguntas não padronizadas, época de aplicação do questionário, ordem das questões, dentre outros, que podem alterar as estimativas, ou seja, as medidas podem variar em função das estratégias utilizadas na coleta das informações ${ }^{5,17}$.

No entanto, este estudo revelou que o inquérito telefônico constitui uma alternativa rápida para disponibilizar estimativas da prevalência das condições crônicas estudadas, apresentando resultados semelhantes aos obtidos pelo inquérito domiciliar. Estudos periódicos comparando dados obtidos do inquérito telefônico aos de pesquisas domiciliares em regiões e localidades com diferentes coberturas, são importantes para avaliar e monitorar a validade do sistema VIGITEL.

Ainda, comparações de estimativas sobre condições crônicas e fatores de risco modificáveis para as doenças crônicas não transmissíveis, obtidas por meio de diferentes modalidades de inquéritos em outras populações, são necessárias para generalização dos achados deste estudo.

\section{Referências}

1. Viacava F. Informações em saúde: a importância dos inquéritos populacionais. Ciênc Saúde Coletiva. 2002;7(4):607-21.

2. Cesar CLG, Barata RB. Editorial Rev Bras Epidemiol. 2008;11Suppl 1:3-5.

3. Lavrakas PJ. Telephone survey methods: sampling, selection and supervision. London: Sage Publications, 1990.

4. Monteiro CA, Moura EC, Jaime PC, Lucca A, Florindo AA, Figueiredo ICR, et al. Monitoramento de fatores de risco para as doenças crônicas por entrevistas telefônicas. Rev Saúde Pública. 2005;39(1):47-57.

5. Nelson DE, Powell-Griner E, Town M, Kovar MG. A comparison of national estimates from the National Health Interview Survey and the Behavioral Risk Factor Surveillance System. Am J Public Health. 2003;93(8):1335-41.

6. Bernal R, Silva NN. Cobertura de linhas telefônicas residenciais e vícios potenciais em estudos epidemiológicos. Rev Saude Publica. 2009;43(3):421-6.

7. The World Health Report, 2002: reducing risks, promoting healthy life. Geneva: World Health Organization , 2002.

8. Brasil. Ministério da Saúde. Secretaria de Vigilância em Saúde. Departamento de Análise de Situação de Saúde. Guia metodológico de avaliação e definição de indicadores: doenças não transmissíveis e Rede Carmem. Brasília: Ministério da Saúde, 2007.

9. Chrestani MA, Santos IS, Matijasevich AM. Hipertensão arterial sistêmica auto-referida: validação diagnóstica em estudo de base populacional. Cad Saúde Pública. 2009;25(11):2395-2406.
10. Barros MBA. Inquéritos domiciliares de saúde: potencialidades e desafios. Rev Bras Epidemiol. 2008;11 Suppl 1:6-19.

11. Cesar CLG, Carandina L, Alves MCGP, Barros MBA, Goldbaum M. Saúde e condição de vida em São Paulo: inquérito multicêntrico de saúde no Estado de São Paulo ISA-SP. São Paulo: USP/FSP; 2005. 212p.

12. Ministério da Saúde. VIGITEL Brasil 2006. Vigilância de fatores de risco e proteção para doenças crônicas por inquérito telefônico: estimativas sobre freqüência e distribuição sócio-demográfica de fatores de risco e proteção para doenças crônicas nas capitais dos 26 estados brasileiros e no Distrito Federal em 2006. Brasília: Ministério da Saúde; 2007.

13. Lwanga SK, Lemeshow S Sample size determination in health studies: a practical manual. Geneva: World Health Organization,1991.

14. Brasil. Ministério da Saúde. Secretaria de Vigilância em Saúde. Secretaria de Gestão Estratégia e Participativa. VIGITEL Brasil, 2008: vigilância de fatores de risco e proteção para doenças crônicas por inquérito telefônico. Brasília: Ministério da Saúde, 2009.

15. Lee S, Davis WW, Nguyen HA, McNeel TS, Brick JM, FloresCervantes I. Examining trends and averages using combined cross-sectional survey data from multiple years. CHIS Methodology Paper, 2007. [cited 2011 Jun 02]. Available from: http://www.chis.ucla.edu/pdf/paper_trends_averages.pdf.

16. Kish L. Survey sampling. New York: John Wiley and Sons; 1965.

17. Fahimi M, Link M, Schwartz DA, Levy P, Mokdad A. Tracking chronic disease and risk behavior prevalence as survey 
participation declines: statistics from the Behavioral Risk Factor Surveillance System and other national surveys. Prev Chronic Dis. 2008;5(3):A80.

18. Cricelli C, Mazzaglia G, Samani F, Marchi M, Sabatini A, Nardi R, et al. Prevalence estimates for chronic diseases in Italy: exploring the differences between self-report and primary care databases. J Public Health Med. 2003;25(3)254-7.

19. Okura Y, Urban LH, Mahoney DW, Jacobsen SJ, Rodeheffer RJ. Agreement between self-reported questionnaires and medical record data was substantial for diabetes, hypertension, myocardial infarction and stroke but not for heart failure. J Clin Epidemiol. 2004;57(10):1096-103.

20. Barros MBA, Cesar CLG, Carandina L, Torre GD. Desigualdades sociais na prevalência de doenças crônicas no Brasil, PNAD-2003. Ciênc Saúde Coletiva. 2006;11(4):911-26.

21. Molenaar EA, Van Ameijden EJ, Grobbee DE, Numans ME. Comparison of routine care self-reported and biomedical data on hipertension and diabetes: results of the Utrecht Health Project. Eur J Public Health. 2007;17(2):199-205.

22. Lima-Costa MF, Peixoto SV, Firmo JOA, Uchoa E. Validade do diabetes auto-referido e seus determinantes: evidências do projeto Bambuí. Rev Saúde Pública. 2007;41(6):947-53.

23. Vargas CM, Burt VL, Gllum RF, Pamuk ER. Validity of self-reported hypertension in the National Health and Nutrition Examination Survey III, 1988-1991. Prev Med. 1997;26(5):678-85.
24. Lima-Costa MF, Peixoto SV, Firmo JOA. Validade da hipertensão arterial auto-referida e seus determinantes (projeto Bambuí). Rev Saúde Pública. 2004;38(5):637-42.

25. Frazão $P$, Naveira M. Prevalência de osteoporose: uma revisão crítica. Rev Bras Epidemiol. 2006;9(2):206-14.

26. Martini LA, Moura EC, Santos LC, Malta DC, Pinheiro MM Prevalência de diagnóstico auto-referido de osteoporose, Brasil, 2006. Rev Saúde Pública. 2009;43Suppl 2:107-16.

27. Hasselgren M, Arne M, Lindahl A, Janson S, Lundbäck B. Estimated prevalences of respiratory symptoms, asthma and chronic obstructive pulmonary disease related to detection rate in primary health care. Scand J Prim Health Care. 2001;19(1):54-7.

28. Mullerova H, Wedzicha J, Soriano JB, Vestbo J. Validation of a chronic obstructive pulmonary disease screening questionnaire for population surveys. Respir Med. 2004;98(1):78-83.

29. Lima-Costa MF, Barreto SM, Giatti L. Condições de saúde, capacidade funcional, uso de serviços de saúde e gastos com medicamentos da população idosa brasileira: um estudo descritivo baseado na Pesquisa Nacional por Amostra de Domicílios. Cad Saúde Pública. 2003;19(3):735-43.

30. Goldman N, Lin IF, Weinstein M, Lin YH. Evaluating the quality of self-reports of hypertension and diabetes. J Clin Epidemiol. 2003;56(2):148-54.

Recebido em: 05/01/2011 Versão final apresentada em: 17/02/2011 Aprovado em: 19/02/2011 